\title{
SCD Inhibition Protects from $\alpha$-Synuclein-Induced Neurotoxicity But Is Toxic to Early Neuron Cultures
}

\author{
Justin W. Nicholatos, ${ }^{1,2}$ Joost Groot, ${ }^{3}$ Shekhar Dhokai, ${ }^{4}$ David Tran, ${ }^{1}$ Lori Hrdlicka, ${ }^{1}$ Thomas M. Carlile, ${ }^{3}$ \\ Melissa Bennion, ${ }^{5}$ Isin Dalkilic-Liddle, ${ }^{4}$ Warren D. Hirst, ${ }^{1}$ and ${ }^{\circledR}$ Andreas Weihofen ${ }^{1}$
}

\section{https://doi.org/10.1523/ENEURO.0166-21.2021}

${ }^{1}$ Neurodegeneration Research Unit, Biogen, Cambridge, MA 02142, ${ }^{2}$ Biogen Postdoctoral Scientist Program, Biogen, Cambridge, MA 02142, ${ }^{3}$ Translational Genome Sciences, Biogen, Cambridge, MA 02142, ${ }^{4}$ Biologics Drug Discovery, Biogen, Cambridge, MA 02142, and ${ }^{5}$ Bioassays and High-Throughput Screens, Biogen, Cambridge, MA 02142

\begin{abstract}
Here, we report the independent discovery and validation of stearoyl-CoA desaturase (SCD) as a modulator of $\alpha$-synuclein ( $\alpha$ Syn)-induced pathology and toxicity in cell-based Parkinson's disease (PD) models. We identified SCD as top altered gene from transcriptional profiling in primary neurons exogenously expressing $\alpha$ Syn with the amplified familial PD mutation 3K. Thus, we sought to further explore SCD as a therapeutic target in neurodegeneration. We report that SCD inhibitors are toxic to early human and rat neuron cultures while displaying minimal toxicity to late cultures. The fatty acid product of SCD, oleic acid (OLA), fully rescues this toxicity in early cultures, suggesting on-target toxicity. Furthermore, SCD inhibition rescues $\alpha$ Syn 3K-induced toxicity in late primary neurons. We also confirm that SCD inhibitors reduce formation of $\alpha$ Syn accumulations, while OLA increases these accumulations in an $\alpha$ Syn 3K neuroblastoma model. However, we identify a caveat with this model where $\alpha$ Syn 3K levels can be suppressed by high SCD inhibitor concentrations, obscuring true effect size. Further, we show that both SCD1 or SCD5 knock-down reduce $\alpha$ Syn 3K accumulations and toxicity, making both a putative drug target. Overall, we confirm key findings of published data on SCD inhibition and its benefits in $\alpha$ Syn accumulation and stress models. The differential neurotoxicity induced by SCD inhibition based on neuron culture age must be accounted for when researching SCD in neuron models and has potential clinical implications. Lastly, our gene profiling studies also revealed novel putative genes connected to $\alpha$ Syn neurotoxicity that are worth further study.
\end{abstract}

Key words: cell-based assays; lipids; neurotoxicity; Parkinson's disease; SCD; synuclein

\section{Significance Statement}

There is no disease-modifying therapeutic for those suffering from Parkinson's disease (PD). Recent research has shown stearoyl-CoA desaturase (SCD) inhibition to ameliorate $\alpha$-synuclein $(\alpha \mathrm{Syn})$-related pathology and neurotoxicity in preclinical PD models. The use of neuronal cell models to study PD-related pathology is critical for developing putative therapeutics. In this work, we demonstrate important caveats in cellular PD models when studying SCD inhibition. We also independently identified SCD and other genes as potential targets for PD. Overall, this work supports SCD as a clinical target and adds important considerations for studying SCD in in vitro models.

\section{Introduction}

As the population becomes increasingly aged it is ever more important to develop therapeutics for age-associated neurodegenerative diseases such as Parkinson's

Received April 7, 2021; accepted July 13, 2021; First published July 22, 2021. All authors are current or former employees of Biogen Inc.
Author contributions: A.W., W.D.H., and J.W.N. designed research; A.W., J.W.N., D.T., L.H., T.M.C., S.D., M.B., I.D.-L., and J.G. performed research; M.B. contributed unpublished reagents/analytic tools; A.W., J.W.N., and J.G. analyzed data; A.W. and J.W.N. wrote the paper.

J. Groot's present address: Inzen Therapeutics, Cambridge, MA, 02139.

L. Hrdlicka's present address: AbbVie, Cambridge Research Center, Cambridge, MA, 02139. 
disease (PD). PD is pathologically characterized by neuronal inclusions, called Lewy bodies (LBs) and Lewy neurites (LNs), and neuronal loss, preferentially of dopaminergic neurons in the substantia nigra pars compacta. LB/LNs are mainly composed of aggregated $\alpha$-synuclein ( $\alpha \mathrm{Syn})$ and lipid membranes (Fares et al., 2021). Reduction of these hallmark structures are considered a therapeutic approach for a disease modifying therapy for PD and other synucleinopathies. Recent studies have shown that inhibition of stearoylCoA desaturase (SCD) ameliorates $\alpha$ Syn-related pathology and neurotoxicity in both in vitro and in vivo models (Vincent et al., 2018; Fanning et al., 2019; Imberdis et al., 2019; Maulik et al., 2019; Terry-Kantor et al., 2020; Nuber et al., 2021). SCD is the rate limiting enzyme in the production of monounsaturated fatty acids (MUFAs), such as oleic acid (OLA). $\alpha$ Syn can directly bind OLA (Sharon et al., 2001) and the presence of OLA in membranes can enhance their interaction with $\alpha$ Syn (Kubo et al., 2005). $\alpha$ Syn's membrane binding properties are believed to play a functional role in synaptic vesicle trafficking and neurotransmission (Runwal and Edwards, 2021). These $\alpha$ Syn-membrane interactions can alter $\alpha$ Syn aggregation kinetics based on lipid membrane composition (Galvagnion et al., 2016). Recent studies have implicated OLA in promoting $\alpha$ Syn-lipid accumulation pathology (Fanning et al., 2019; Imberdis et al., 2019). Altering cellular lipid profiles, and more specifically lowering MUFAs such as OLA through SCD inhibition, may thus be a viable therapeutic approach to target $\alpha$ Syn pathology in PD. Yumanity Therapeutics is testing a SCD inhibitor in clinical trials for PD and has another SCD inhibitor in their pipeline for LB dementia (Yumanity Therapeutics, 2021). Scientists from Yumanity initially discovered SCD inhibition as protective from $\alpha$ Syn-induced toxicity in yeast (Vincent et al., 2018).

Many of the studies demonstrating benefits of SCD inhibition in PD used an "amplified" familial $\alpha$ Syn E46K model, which harbors E35K + E46K + E61K mutations $(\alpha$ Syn $3 K)$. This model is advantageous in its ability to recapitulate features of human PD pathology (Ericsson et al., 2021). Transgenic mice with $\alpha$ Syn $3 \mathrm{~K}$ display dopaminergic neurodegeneration and develop PD like motor symptoms that are responsive to L-Dopa treatment (Nuber et al., 2018). LBs are not only loaded with $\alpha$ Syn but are full of membranous lipids and vesicular structures (Shahmoradian et al., 2019). Transgenic mice and cellular models that express $\alpha$ Syn 3K recapitulate this lipid rich vesicular clustering during accumulation formation and exhibit cytotoxicity (Dettmer et al., 2017; Nuber et al., 2018; Terry-Kantor et al., 2020). E35K, E46K, and E61K $\alpha$ Syn mutations alone and in combination have been

Acknowledgements: We thank members of Neurodegeneration Research Unit's Movement Disorders group for critical project feedback and Naomi Okugawa for generating NGN2 neurons.

Correspondence should be addressed to Andreas Weihofen at andreas. weihofen@biogen.com.

https://doi.org/10.1523/ENEURO.0166-21.2021

Copyright (C) 2021 Nicholatos et al.

This is an open-access article distributed under the terms of the Creative Commons Attribution 4.0 International license, which permits unrestricted use, distribution and reproduction in any medium provided that the original work is properly attributed. shown to have altered lipid binding properties (Rovere et al., 2019), which likely contributes to their pathogenicity.

Here, we report the independent discovery of SCD as a top hit identified in a transcriptional profiling study investigating $\alpha$ Syn neurotoxicity. This internal discovery and the active clinical interest spurred us to validate key published findings and expand data on SCD inhibition in PD models. In addition to supporting much of the published data on SCD inhibition in cell-based models, we also identified important caveats that must be considered when studying this target in neurons and $\alpha$ Syn overexpressing systems.

\section{Materials and Methods}

\section{Antibodies}

Antibodies used were MJFR1 (Abcam, 138501) or syn1/Clone 42 (Biosciences, 610787) for total $\alpha$ Syn (Abcam, 138501), EP1536Y for pSer129 $\alpha$ Syn (Abcam, ab51253), MJFR14-6-4-2 (Abcam, 209538) for oligomeric/fibrillar $\alpha$ Syn, C4 for loading control actin (Abcam, ab14128), and CD.E10 for SCD (ThermoFisher Scientific, MA5-27542).

\section{SCD inhibitors and OLA}

MF-438 (MF; EMD Millipore, 569406) and CAY10566 (CAY; Ambeed, A698046) were resuspended in DMSO and dispensed to cell cultures via a HP D300e digital dispenser. OLA BSA conjugate (O3008-5ML) and fatty acidfree BSA control (A8806) were purchased from Sigma. OLA and BSA control were diluted in culture media before addition to cell culture ( $50 \%$ exchange).

\section{AAV generation and transduction}

cDNAs for human $\alpha$ Syn, $\alpha$ Syn 3K $(\alpha$ Syn E35K + E46K + E61K) and $\alpha$ Syn 3K S129A were synthesized (Geneart, ThermoFisher Scientific) and subcloned into AAV entry vectors under the CAG promoter. Empty vector without insert control (EV) vectors were packaged into adeno-associated viruses (AAVs) using AAV9 capsid serotype (Packgene Biotech). Control AAV (EV) was generated using entry vector without cDNA insert. Purity was determined using SDS-PAGE followed by Coomassie staining. AAV genome copies were quantified by determining copy numbers of AAV2 ITRs via qRT-PCR. Cells were transduced at a multiplicity of infection (MOI) of $80 \mathrm{~K}$ for primary cortical neuron cultures and 250K for iPSC neurons, unless stated otherwise.

\section{Primary neuron cultures}

Mouse cortical neuron cultures were isolated and homogenized from timed-pregnant C57BL/6J mouse embryos on embryonic day (E)17 according to Institutional Animal Care and Use Committee (IACUC) guidelines. Rat cortical neurons cultures were generated from timed pregnant Sprague Dawley female rats at gestational day E16. Briefly, cortex tissues were dissected on ice in HBSS without calcium or magnesium (Invitrogen, catalog \#21022-CV), carefully separated from the meninges, washed $3 \times$ in ice-cold HBSS, and then incubated at $37^{\circ} \mathrm{C}$ with $0.25 \%$ Trypsin-EDTA and $1 \mathrm{mg} / \mathrm{ml}$ DNase I (Sigma, 
catalog \#DN25-10MG) for $15 \mathrm{~min}$. Cell were resuspended in Neurobasal media (Invitrogen, catalog \#21103) containing $10 \%$ fetal bovine serum (FBS) and $1 \times$ GlutaMAX (Invitrogen, catalog \#35050) and filtered through a 100- $\mu \mathrm{m}$ cell strainer. Neurons were plated at a density of 16,000 (imaging) or 50,000 (CellTiter-Glo) cells per well in Corning Biocoat Poly-D-Lysine 96-well plate Cellware (ThermoFisher Scientific, catalog \#356640) with black wells and clear bottom with lid; $1.5 \mathrm{~h}$ later, plating media were aspirated and replaced with neurobasal feeding media supplemented with 2\% B27 supplement (Invitrogen, catalog \#17504-044), 1× GlutaMAX, and $1 \times$ pen/strep (Invitrogen, catalog \#15140122).

SCD1 inhibitors, OLA, and AAVs were given on day in vitro (DIV)7 for early cultures and DIV18 for late cultures. OLA and AAVs were added with fresh media. Cultures were fixed (4\% PFA in PBS for $15 \mathrm{~min}$ ) for imaging or lysed for analysis $12 \mathrm{~d}$ after treatments.

\section{RNA sequencing (RNA-Seq)}

Rat cortical neuron cultures were treated with AAV9 EV or AAV9- $\alpha$ Syn 3K on DIV5. RNA was isolated 6, 12, and $19 \mathrm{~d}$ after AAV treatment with a RNeasy plus kit with DNase digestion. RNA quality was measured using the RNA 6000 Pico kit (Agilent, 5067-1513) on a Bioanalyzer 2100 , all RNA integrity scores were $\geq 9$. There were five replicates per treatment and for every time point (30 samples total). cDNA was prepared with the SMART-Seq v4 Ultra Low Input RNA kit (Takara, 634891) according to manufacturer's instructions. A total of $8 \mathrm{ng}$ of input RNA with $1.6 \mu \mathrm{l}$ of added 1:10,000 ERCC RNA (ThermoFisher, 4456740) was used for cDNA generation, followed by cDNA amplification with seven cycles of PCR using CB PCR Buffer (Takara, 638526). Amplified cDNA was quantified using the High Sensitivity DNA kit (Agilent, 50674626) on a Bioanalyzer 2100. Libraries were prepared from amplified cDNA using the Nextera XT DNA Library Prep kit (Illumina, FC-131-1096) according to manufacturer's instructions; $150 \mathrm{pg}$ of cDNA was used as input, 12 cycles of PCR were used to amplify tagmented cDNA with Nextera Index kit Set A (Illumina, FC-131-2001), and library cleanup was performed as recommended for 300- to 500-bp libraries. Libraries were quantified and QCed on the Lab Chip GX DNA HS Chip (PerkinElmer, CLS760672). Libraries were normalized to $10 \mathrm{~nm}$, pooled, and sequenced on a HiSeq 2500 in paired end mode $2 \times 50$ $\mathrm{bp}$ to an average depth of 8.8 million reads per sample.

RNA-Seq read data were analyzed using a pipeline that used STAR (version 2.5.2b; Dobin et al., 2013) for read alignment and RSEM (version 1.2.31; Li and Dewey, 2011) for transcript read count quantification on the genome (Rattus norvegicus.Rnor_6.0.89) with $\alpha$ Syn 3K vector added. All samples passed quality checks on raw reads with FASTQC (Wingett and Andrews, 2018) and read alignment quality ( $>90 \%$ uniquely mapped paired reads). Normalization and differential expression analysis were conducted with the Bioconductor package DESeq 2 (Love et al., 2014) using a statistical model formulated with time and treatment factors. Sample expression variation was quantified using PCA on gene TPM data which showed sample clustering per factor (time, treatment, no outliers). Thresholds of false discovery rate (FDR) $<0.05$ were consistently applied to identify differentially expressed genes. To identify differential expression changes consistent across $\alpha$ Syn 3K treatments and across time points, a rank-based meta-analysis was performed with Rankprod (Del Carratore et al., 2017). Rat genes were mapped to the mouse genome according to the orthologous gene mapping from Ensembl biomart for analysis. Pathway enrichment analysis was performed using Fisher exact tests using the Reactome database. RNA-Seq data were deposited at the Gene Expression Omnibus accession \#GSE172385

\section{Neuroblastoma M17 $\alpha$ Syn 3K-GFP model}

Human full-length $\alpha$ Syn 3K with C-terminal GFP ( $\alpha$ Syn 3K-GFP) was cloned into pLVX-TetOne-Puro lentiviral vector (Clontech 631849). $\alpha$ Syn 3K-GFP lentivirus was produced in 293T cells by co-transfecting the lentiviral plasmid and lentiviral packaging mix (Virapower, Thermo Scientific) using Lipofectamine 2000 (Invitrogen 11668$500)$. Stable inducible BE(2)-M17 cells were generated by three consecutive rounds of lentiviral spinfection and selection in complete growth medium containing $1 \mu \mathrm{g} / \mathrm{ml}$ puromycin (Invitrogen A1113803). After third round of transduction, cells were selected in complete growth medium containing $10 \mu \mathrm{g} / \mathrm{ml}$ puromycin and single-cell clones were generated by limiting dilution. $\alpha$ Syn 3K-GFP expression and accumulation formation was induced by addition of $2 \mu \mathrm{g} / \mathrm{ml}$ doxycycline to the culture medium. Multiple single-cell clones were assessed by counting the GFP positive cells and then quantifying the percentage of cells with accumulations. This was accomplished by high-content imaging using the Opera Phenix. The line with the most accumulations was chosen for subsequent assays.

Neuroblastoma cells were cultured in 50\% EMEM (ATCC 30-2003), 50\% Ham's F-12 (Invitrogen 11765-054), and $10 \%$ FBS, tetracycline free (Clonetech 631101). Media were supplemented with 1\% Glutamax (Invitrogen 35050061), 1\% MEM non-essential amino acids (Invitrogen 1140-050), and penicillin-streptomycin; $1 \mu \mathrm{g} / \mathrm{ml}$ puromycin was used in the stock media for $\alpha$ Syn 3K-GFP selection.

$\alpha$ Syn 3K-GFP accumulations were assessed after 48-h induction with doxycycline $(2 \mu \mathrm{g} / \mathrm{ml})$ by counting the number of cells that were positive, giving a \% of cells that harbored accumulations. This was accomplished with live imaging using PerkinElmer Opera Phenix high-content screening system.

\section{iPSC neuron culture}

Human iPSC source was a Lymphoblastoid Cell Line ND07189 obtained from Coriell Cell Repository. The donor is a non-Hispanic/Latino, white male (46 XY). iPSCs were generated using the Epi5 Episomal iPSC Reprogramming kit (ThermoFisher Scientific). iPSCs were confirmed to be (1) grossly karyotypically normal (46 XY); (2) pluripotent; (3) myoplasma and human pathogen-free (except for EBV + ). Neurons were differentiated from 7189L-NGN2-H2 iPSCs which carry a tet-inducible NGN2 expression cassette 
targeted into the AAVS1 safe harbor locus. Three days after doxycycline treatment to induce NGN2 expression and differentiation, neurons were frozen.

Previously frozen NGN2 neurons were partially thawed in $37^{\circ} \mathrm{C}$ water bath. The exterior of vial was sterilized with $70 \% \mathrm{EtOH}$, then placed inside the TC hood. Cells were transferred to sterile 50-ml tubes, and slowly resuspended with $5 \mathrm{ml}$ of room temperature NB/B27 plating media [Neurobasal:DMEM/F12 (1:1 vol) $+200 \mu \mathrm{M}$ ascorbic acid +1 $\mu \mathrm{m} \mathrm{dbcAMP}+10 \mathrm{ng} / \mathrm{ml} \mathrm{BDNF}+10 \mathrm{ng} / \mathrm{ml} \mathrm{GDNF}+1 \times$ pen/ strep $+1 \mu \mathrm{g} / \mathrm{ml}$ doxycycline + CultureOne]. Cells were spun down at $800 \mathrm{rpm}$ for $4 \mathrm{~min}$ at room temperature, and cell pellet was gently resuspended in NB/B27 plating media. Cells were counted on Vi-Cell and plated on PDL-coated microplates. To minimize edge effect, microplates were left for 30 min inside the TC hood to allow cells to attach, before placing inside the $37^{\circ} \mathrm{C} / \mathrm{CO}_{2} \mathrm{TC}$ incubator. Doxycycline treatment continued until DIV7, at which point half the media were exchanged with [Neurobasal:DMEM/F12 (1:1 vol) + $200 \mu \mathrm{m}$ ascorbic acid $+1 \mu \mathrm{m}$ dbcAMP $+20 \mathrm{ng} / \mathrm{ml} \mathrm{BDNF}+20 \mathrm{ng} / \mathrm{ml}$ GDNF + $1 \times$ pen/strep].

SCD inhibitor, AAV, and or OLA treatments began on DIV7 and DIV21 for early and late cultures, respectively.

\section{siRNA treatment}

siRNAs were procured from Integrated DNA Technologies: hs.Ri.SCD.13.1, hs.Ri.SCD.13.2, hs.Ri.SCD.13.3, hs.Ri. SCD5.13.1, hs.Ri.SCD5.13.2, hs.Ri.SCD5.13.3. Three siRNAs were pooled in equal amounts for each target. siRNAs were delivered to neuroblastoma cells in Opti-MEM (Invitrogen) using Lipofectamine RNAiMAX (Invitrogen, 13778). siRNA treatments were given $24 \mathrm{~h}$ before doxycycline induction.

\section{Cell viability measurements}

Cytotoxicity in neuroblastoma M17 $\alpha$ Syn 3K-GFP model was assessed by caspase 3 and 7 activity via a commercial kit (Promega, G8090). Before lysis and caspase measurement cell counts were taken by staining with Hoechst and Opera Phenix high-content imaging. Caspase activity was normalized to cell counts. Viability in rodent cortical neurons and in NGN2 neurons was assessed by CellTiter-Glo (Promega, G7571). In addition, neuronal and astrocytic viability in rat cortical neurons was determined after fixation, permeabilization $(0.1 \%$ Triton X-100 for $20 \mathrm{~min}$ ), and staining with MAP2 (Abcam, ab92434) and GFAP (Abcam, ab7260) by neuron and astrocyte counting in the Opera Phenix high-content imaging system. MAP2 and GFAP were visualized by Alexa Fluor 647 and 488 secondary antibodies.

\section{qRT-PCR}

mRNA isolation was conducted one of two ways. For primary and iPSC neurons, the Cells-to-CT kit was used for mRNA isolation and cDNA synthesis (TaqMan Fast Advanced Cells-to-CT kit, A35374). For neuroblastoma cells, the Cells-to-CT kit was used or the combination of the RNeasy Plus Micro kit (QIAGEN, 74134) with iScript Reverse Transcription Supermix for RT-qPCR (Bio-Rad, 1708840) for mRNA isolation and cDNA synthesis. qRT-
PCR was then performed using the TaqMan Fast Advanced Master Mix (ThermoFisher Scientific, 4444556) with QuantStudio 7 Pro Real-Time PCR System.

For qRT-PCR, target genes were normalized and multiplexed with HPRT gene expression (neuroblastoma and iPSC neurons) or with ACTB (rat primary neurons). Reference genes used VIC and target genes FAM dye signal.

TaqMan primer list: HPRT1, Hs02800695_m1; SCD, Hs01682761_m1; SCD5, Hs00227692_m1; SNCA, Hs00240906_m1; ACTB, Rn00667869_m1; SCD1, Rn06152614_s1; and SNCA, Rn01425140_m1.

\section{Human tissue}

Frozen postmortem amygdala tissues of a non-demented control donors (female, 70 years of age) and a donor with clinical diagnosis of PD (male, 87 years of age, Braak stage 6) were obtained from the Netherlands Brain Bank (NBB; Netherlands Institute for Neuroscience, Amsterdam, open access; www.brainbank.nl). Written informed consent for the use of the samples for research purposes and clinical information of donors was obtained by the NBB.

\section{Western blotting and dot blot analysis}

Cells were lysed in RIPA buffer with protease and phosphatase inhibitors. Lysates were protein normalized using BCA assay. Where indicated proteins were sequentially extracted from tissue or cells in high salt $(750 \mathrm{~mm}), 1 \%$ Triton X-100 buffer and 1\% SDS buffer as described previously (Weihofen et al., 2019). For Western blotting, proteins were then resolved on Bolt gels $(4-12 \%)$ under reducing conditions using MES SDS running buffer system, followed by transfer to PVDF membrane with the iBlot $2 \mathrm{Gel}$ Transfer system. For dot blot analysis, total extracts were spotted onto $0.45-\mu \mathrm{m}$ nitrocellulose membranes (normalized for total $\alpha$ Syn expression). Membranes were blocked in either $5 \%$ milk in TBS $(0.05 \%$ Tween) or LICOR blocking buffer $(0.05 \%$ Tween 20) and probed with primary antibodies diluted in respective blocking buffer. Bound antibodies were detected by enhanced chemiluminescence or LICOR infrared imaging system.

\section{Statistical analysis}

Analyses were performed with GraphPad Prism 9. Significant level was defined as $p<0.05$. Intergroup differences were tested using two-tailed $t$ test, one-way ANOVA or two-way ANOVA. Multicomparisons were corrected with Dunnett's post hoc test when comparing three or more groups (for details, see Extended Data Fig. 1-2). Data were displayed as boxplot or line charts showing error bars with SDs. Statistical analyses for RNA-Seq data are described in its own section above.

\section{Results}

To reveal mechanistic insights into $\alpha$ Syn neurotoxicity, we performed gene expression profiling on neurons overexpressing cytotoxic $\alpha$ Syn. To do so, we first overexpressed human wild-type $\alpha$ Syn ( $\alpha$ Syn WT) and $\alpha$ Syn 
A

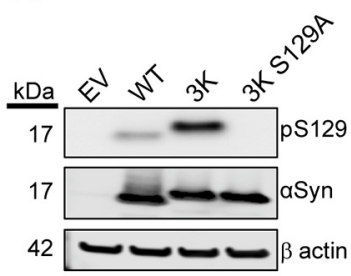

D

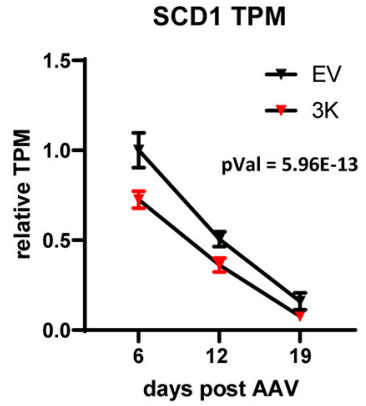

B

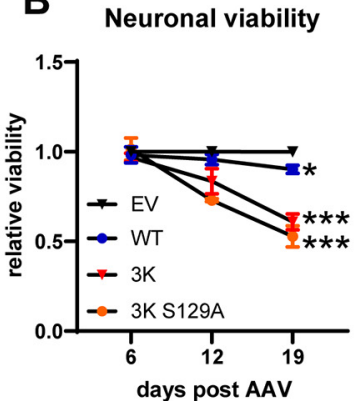

E

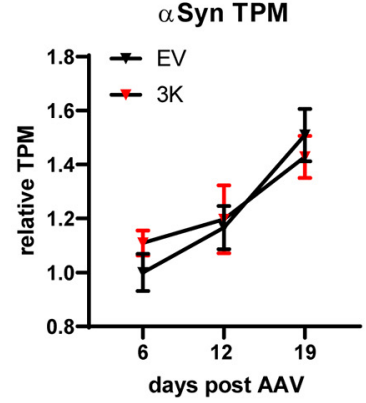

C

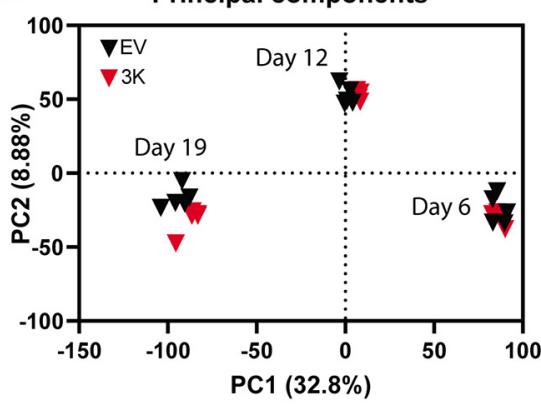

$\mathbf{F}$

SCD1 mRNA

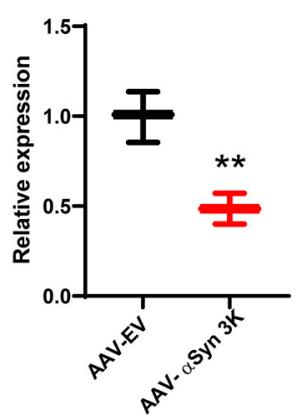

Figure 1. RNA-Seq identifies SCD as a $\alpha$ Syn $3 \mathrm{~K}$ neurotoxicity regulated gene in rat cortical neuron cultures. $\boldsymbol{A}$, Western blotting showing equivalent expression of $\alpha$ Syn WT, $\alpha$ Syn 3K, or $\alpha$ Syn 3K S129 phosphorylation mutant (S129A) in rat cortical neuron cultures on respective AAV transduction. $\boldsymbol{B}$, Viability assessed by ATP levels (CellTiter-Glo) in rat cortical neuron cultures under respective AAV9 transduction, two-way ANOVA with Dunnett's multiple test correction. Dose-dependent and time-dependent toxicity analysis in mouse and rat cortical neuron cultures are shown in Extended Data Figure 1-1. C, Principal component analysis of RNASeq data from rat cortical neurons with AAV9-EV or AAV9- $\alpha$ Syn 3K $(6,12$, and $19 \mathrm{~d}$ after AAV9 transduction). $\boldsymbol{D}$, Relative transcripts per million of SCD1 transcripts in AAV9-EV and AAV9- $\alpha$ Syn 3K samples. $E$, Relative transcripts per million of endogenous rat SNCA ( $\alpha$ Syn) transcripts in AAV9-EV and AAV9- $\alpha$ Syn 3K samples. $\boldsymbol{F}$, qPCR confirmation of SCD1 transcript suppression from AAV9- $\alpha$ Syn $3 \mathrm{~K}$ in rat cortical neuron cultures $12 \mathrm{~d}$ after AAV9 transduction, two tailed $t$ test. Data displayed as boxplots or as line charts showing error bars with SD; ${ }^{*} p<0.05,{ }^{* *} p<0.01,{ }^{* * *} p<0.001$. Extended Data Figure 1-2 displays additional information on statistical tests applied in present study.

$3 \mathrm{~K}$ in rat primary cortical neuron cultures using AAV9. Equivalent overexpression was confirmed by Western blotting for $\alpha$ Syn WT and 3K (Fig. 1A). Cell viability was assessed by measuring ATP levels and was significantly reduced by $\sim 40 \% 19 \mathrm{~d}$ after AAV9 $\alpha$ Syn 3K compared with EV AAV9 transduction (Fig. 1B). A clear trend toward cytotoxicity was observed after 12 but not $6 \mathrm{~d}$ with $\alpha$ Syn $3 \mathrm{~K}$ (Fig. 1B). In contrast, only minor or no cytotoxicity was observed with $\alpha$ Syn WT in this system (Fig. 1B). Dose-dependent and time-dependent cytotoxicity was observed in rat and mouse cortical neuron cultures, where mouse cultures showed greater sensitivity to $\alpha$ Syn 3K stress (Extended Data Fig. 1-1). Of note, we found $\alpha$ Syn $3 \mathrm{~K}$ to be hyperphosphorylated at $\mathrm{S} 129$ in comparison to $\alpha$ Syn WT, and mutation of the phosphorylation site (S129A) did not reduce cytotoxicity (Fig. 1A, $B)$. This suggests that phosphorylation at $S 129$ is not required for the cytotoxic properties of $\alpha$ Syn 3K.

Having found $\alpha$ Syn $3 \mathrm{~K}$ to elicit robust toxicity in neuron cultures we then performed RNA-Seq on rat cortical neuron cultures treated with AAV9 $\alpha$ Syn $3 \mathrm{~K}$ or EV with isolations at 6,12 , and $19 \mathrm{~d}$ after AAV transduction. Principal component analysis of the gene expression profiling in our primary rat neuron cultures showed the greatest transcriptional changes between different culture ages $(6$ vs 12 vs $19 \mathrm{~d}$ after AAV9; Fig. 1C). Furthermore, the expression of endogenous $\alpha$ Syn increases in the neuron cultures through time (Fig. 1E), which has been reported in primary neuron maturation previously (Courte et al., 2020). The PCA and $\alpha$ Syn expression data combined suggest that our primary cultures are maturating in vitro. Separation of EV and $\alpha$ Syn $3 \mathrm{~K}$ groups began at $12 \mathrm{~d}$ after transduction (Fig. 1E). Table 1 shows a list with the top ten significantly changed genes when including all three time points. We focused on gene hits found in all three time points to ensure robustness of the hits and to uncover pathologic changes occurring before obvious cytotoxicity was observed. Many of these hits were lipid regulatory genes, and indeed pathway analysis showed enrichment in lipid processes and fatty acid metabolism (Extended Data Table 1-2). Among these top hits was SCD1, which was downregulated by $\alpha$ Syn 3K (Fig. 1D). We also confirmed SCD1 downregulation by $\alpha$ Syn 3K by qPCR (Fig. 1F). The SCD2 isoform, which has high expression in the rodent brain, was also down regulated by $\alpha$ Syn 3K (Extended Data Fig. 1-1E; Kaestner et al., 1989).

Next, we wanted to assess the effects of SCD manipulation on $\alpha$ Syn accumulation formation and cytotoxicity. 
Table 1: Top 10 differentially regulated genes by $\alpha$ Syn $3 K$ in primary neuron cultures

\begin{tabular}{llll}
\hline Rank & Gene name & log2FC & Combined FDR $p$ value \\
\hline 1 & Lpcat1 & 0.84 & $2.64 \mathrm{E}-86$ \\
2 & Rnf145 & 0.61 & $5.14 \mathrm{E}-35$ \\
3 & Rgs4 & 0.44 & $2.02 \mathrm{E}-17$ \\
4 & Scd1 & -0.67 & $5.96 \mathrm{E}-13$ \\
5 & Fam102b & 0.84 & $8.20 \mathrm{E}-12$ \\
6 & Acsbg1 & -0.25 & $1.34 \mathrm{E}-10$ \\
7 & Snn & 0.45 & $5.31 \mathrm{E}-10$ \\
8 & Psat1 & -0.29 & $1.34 \mathrm{E}-09$ \\
9 & Rgcc & -0.4 & $1.07 \mathrm{E}-08$ \\
10 & Pcdh8 & 0.26 & $2.01 \mathrm{E}-08$ \\
\hline
\end{tabular}

A meta-analysis was performed to identify the top differentially regulated genes at 6,12 , and $19 \mathrm{~d}$ after AAV9 transduction time points. These are the top 10 genes from this meta-analysis (see Extended Data Table 1-1 for the full results, and Extended Data Table 1-2 for pathway analysis); $p$ values are corrected for false discovery (for details, see Materials and Methods).

To do so, we developed a M17 human neuroblastoma cell model with doxycycline-inducible $\alpha$ Syn 3K-GFP expression. Similar $\alpha$ Syn 3K models, which display cytotoxicity and form lipid rich $\alpha$ Syn accumulations that are a potential early pathogenic step toward LBs, had previously been developed in the same neuroblastoma cell line (Dettmer et al., 2017; Imberdis et al., 2019; Terry-Kantor et al., 2020). To highlight differences between LBs and $\alpha$ Syn 3K accumulations, we show that pS129 $\alpha$ Syn is mostly soluble in our neuroblastoma $\alpha$ Syn $3 \mathrm{~K}$ model while it is almost exclusively Triton X-100 insoluble and displays a ladder-like pattern on SDS PAGE in PD patient brain (Extended Data Fig. 2-2A,B). Similarly, no signal for oligomeric/fibrillar $\alpha$ Syn was detected in a dot blot analysis with $\alpha$ Syn 3K neuroblastoma extract (Extended Data Fig. $2-2 C)$. For quantifying $\alpha$ Syn 3K-GFP accumulations we applied high-content imaging and used caspase 3/7 activation for assessing $\alpha$ Syn 3K-induced cytotoxicity. $\alpha$ Syn $3 \mathrm{~K}$ accumulations were reduced by $\sim 80 \%$ if cells where fixed in presence of $1 \%$ Triton X-100, showing that accumulations are not composed of fibrillar $\alpha$ Syn (Extended Data Fig. 2-2D,E). We then recapitulated a dose-dependent increase in accumulation formation from treatment with the SCD product OLA (Fig. 2A,H; Fanning et al., 2019). We also found that the formation of accumulations coincides with increased caspase $3 / 7$ activity and that OLA treatment can further increase it (Fig. 2B).

Humans have two SCD isoforms (SCD1 and SCD5), and we tested whether their suppression influenced accumulation formation with siRNA knock-down. mRNA reduction of both isoforms was robust $(\sim 90 \%)$ and in the case of SCD1 led to a near complete loss of protein (Extended Data Fig. 2-1B-D). No antibody for measuring SCD5 protein levels is readily available. siRNA knockdown of both SCD1 and SCD5 led to a suppression of accumulations, albeit to a lesser degree by SCD5 knockdown ( $20 \%$ vs $10 \%$ suppression; Fig. $2 C$ ). Of note, SCD5 is expressed $\sim 80 \%$ less than SCD1 in our neuroblastoma model but still influenced accumulation formation (Fig. 2D). SCD1 knock-down also rescued $\alpha$ Syn 3K-GFP accumulation-induced toxicity as assessed by caspase 3/7 activity (Extended Data Fig. 2-1A). We also recapitulated a dose-dependent decrease in accumulations from the SCD inhibitors CAY and MF (Fig. 2E,F,H). SCD inhibition suppressed accumulations prophylactically and also therapeutically as when inhibitors were given after accumulation formation (Extended Data Fig. $2-1 F$ ). The observed decreases in accumulations at higher concentrations were much larger compared with complete knock-down of SCD1 or SCD5 by siRNA. At lower concentrations, the reduction of accumulations from SCD inhibition was reestablished by OLA treatment (Fig. 2E,F,H). Moreover, SCD inhibitors also reduced $\alpha$ Syn $3 \mathrm{~K}$-induced caspase $3 / 7$ activation, and OLA reestablished caspase $3 / 7$ activity too at lower concentrations (Fig. 2G).

Interestingly, at higher concentrations of SCD inhibition (1 and $10 \mu \mathrm{M}$ ) we observed that OLA did not reestablish $\alpha$ Syn 3K-GFP-induced accumulations or caspase 3/7 activity (Fig. 2E-G), suggesting off-target effects. To explore this further we measured mRNA and protein levels of $\alpha$ Syn 3K-GFP with CAY and MF inhibition from 0.01 to 10 $\mu \mathrm{M}$. We discovered that at $\geq 1 \mu \mathrm{m}$, there was a dose-dependent suppression of $\alpha$ Syn 3K-GFP mRNA and protein (Fig. $3 A-C$ ), $1 \mu \mathrm{m}$ produced a $\sim 50 \%$ reduction and $10 \mu \mathrm{m}$ a $\sim 90 \%$ reduction. In contrast to exogenous $\alpha$ Syn 3KGFP, endogenous $\alpha$ Syn was unaffected by SCD inhibition (Extended Data Fig. 3-1A). Additionally, robust siRNA knock-down of SCD1 or SCD5 did not alter $\alpha$ Syn 3K-GFP protein levels, suggesting it is not a specific phenomenon related to SCD inhibition (Extended Data Fig. 2-1D,E). Overall, we validate that SCD inhibition lowers $\alpha$ Syn 3K accumulations and reduces $\alpha$ Syn 3K-induced cytotoxicity but add caution to any users of this type of $\alpha$ Syn 3K-GFP model (or similar overexpression system) as high inhibitor concentrations suppressed exogenous mRNA which led to off-mechanism (non-MUFA related) lowering of $\alpha$ Syn 3K accumulations.

Next, we wanted to assess the effects of SCD manipulation in primary neuron cultures. We treated 7 DIV cultures with 0.01 and $0.1 \mu \mathrm{M}$ SCD inhibitors and assessed viability with ATP levels $12 \mathrm{~d}$ after treatment. We found that DIV7 cultures showed dose-dependent cytotoxicity from both CAY and MF compounds (Fig. $4 A, C), 0.01 \mu \mathrm{M}$ led to a $\sim 50 \%$ drop and $0.1 \mu \mathrm{m}$ a $\sim 75 \%$ drop in viability. Importantly, the SCD inhibitor-induced toxicity was fully rescued by treatment with OLA, suggesting the cytotoxicity is on-mechanism. The finding of SCD inhibitor-induced toxicity in primary neurons was surprising as one study showed tolerance of SCD inhibitors on primary rat neurons (Fanning et al., 2019). SCD1 expression decreases overtime in these cultures suggesting that they become less dependent on SCD and OLA as they mature (Fig. 1D). To further explore SCD inhibitor toxicity we repeated the experiment starting at DIV18, hypothesizing more established cultures may have increased tolerance. Indeed, we found no significant SCD inhibitor-induced cytotoxicity in these late cultures (Fig. 4B,C). To confirm that neuron death was occurring in early DIV7 cultures with SCD inhibition, we repeated the viability experiment and counted neurons and astrocytes present in these cultures by staining with MAP2 and GFAP markers, respectively. We found that both neurons and astrocytes were dying from 
A

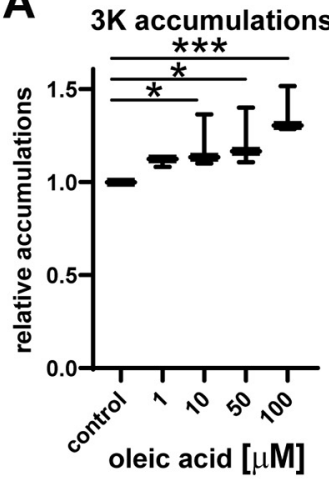

E

3K accumulations CAY

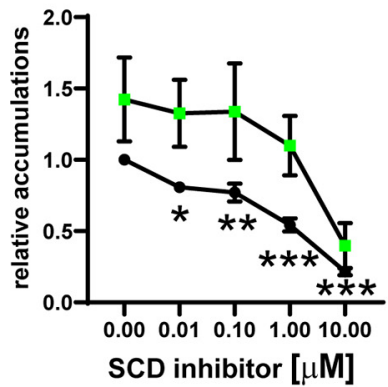

H

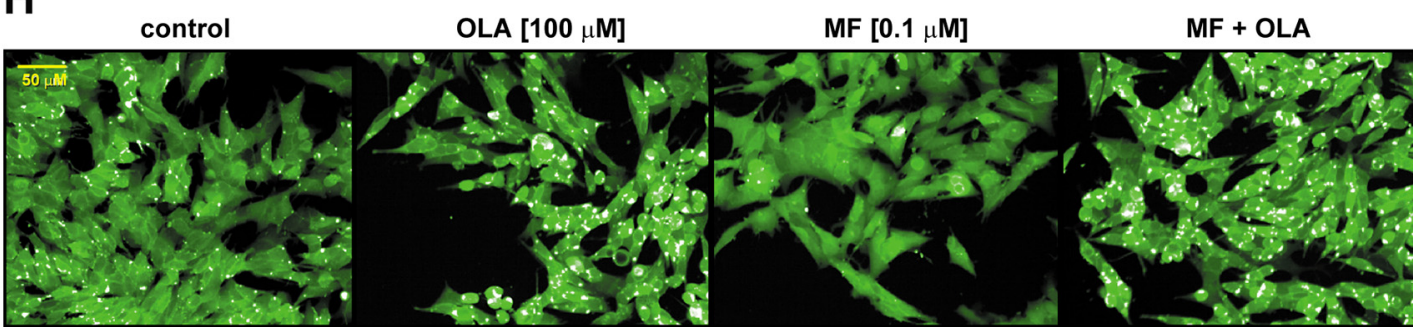

B Caspase 3/7 activity

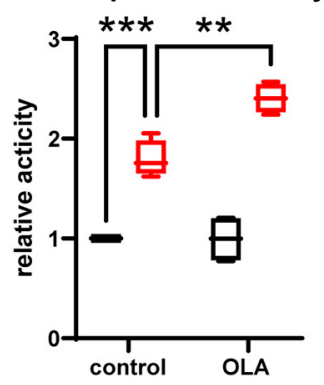

$\mathbf{F}$

3K accumulations MF

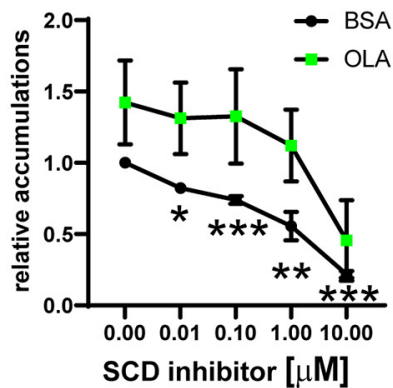

C $3 \mathrm{~K}$ accumulations

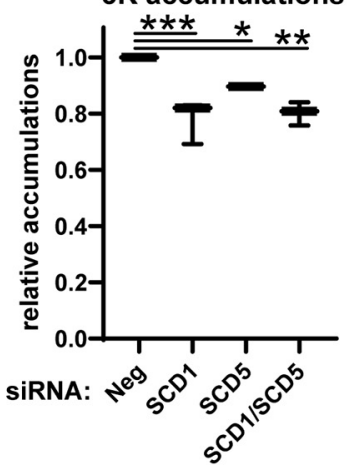

D SCD1 SCD5 mRNA

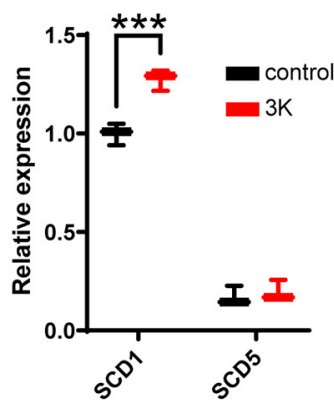

G

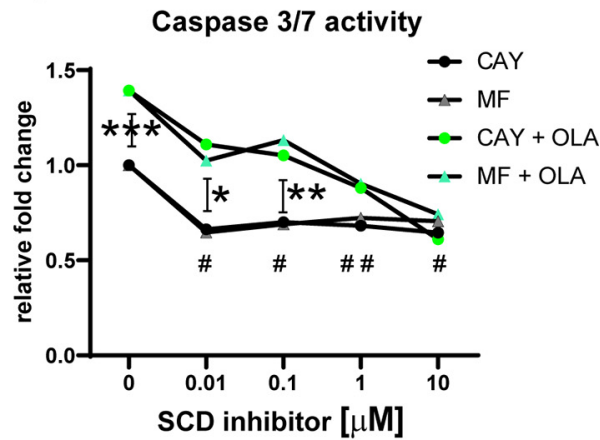

$\alpha$ Syn 3K accumulations

Figure 2. Inhibition of SCD in $\alpha$ Syn 3K-GFP neuroblastoma model reduces accumulations and cytotoxicity. $\boldsymbol{A}$, Dose-dependent increase in $\alpha$ Syn 3K-GFP accumulations from 48-h OLA treatment (1-100 $\mu \mathrm{m})$. B, 48-h induction of $\alpha$ Syn 3K-GFP leads to caspase 3/ 7 activation and $100 \mu \mathrm{m}$ OLA treatment further increases it, whereas SCD1 siRNA reduces it (Extended Data Fig. 2-1A) C, 5 nM siRNA knock-down of SCD1 or SCD5 for $48 \mathrm{~h}$ decreases the number of cells with accumulations. Please see Extended Data Figure $2-1 B-E$ for further siRNA knock-down characterization. $\boldsymbol{D}$, mRNA levels of SCD1 and SCD5 in our neuroblastoma model showing SCD5 has $\sim 80 \%$ less expression than SCD1 and that accumulations can increase SCD1 levels. $\boldsymbol{E}$, Relative fraction of cells with accumulations under increasing concentration of CAY for $48 \mathrm{~h}$ with and without $100 \mu \mathrm{m}$ OLA. $\boldsymbol{F}$, Relative fraction of cells with accumulations under increasing concentration of MF for $48 \mathrm{~h}$ with and without $100 \mu \mathrm{M}$ OLA. Both prophylactic and therapeutic treatment paradigms (Extended Data Fig. 2-1F) reduce accumulations. G, Relative $\alpha$ Syn 3K-GFP accumulation-induced caspase activity with increasing concentration of CAY or MF with and without $100 \mu \mathrm{M}$ OLA for $48 \mathrm{~h}$ (* denotes comparison between non-OLA and OLA treatments, \# denotes respective inhibitor concentration vs DMSO control). Note that OLA does not reestablish the $\alpha$ Syn $3 \mathrm{~K}$ accumulation-induced caspase activity under SCD inhibition $\geq 1 \mu \mathrm{M}$. $\boldsymbol{H}$, Representative high-content images of $\alpha$ Syn 3K-GFP neuroblastoma model with induced $\alpha$ Syn 3K accumulations showing OLA-induced increase, SCD inhibitor-induced decrease, and OLA rescue of accumulations from SCD inhibition. Additional $\alpha$ Syn 3K accumulation characterization including comparison to LBs is shown in Extended Data Figure 2-2. One-way or two-way ANOVA run with Dunnett's multiple test correction, data displayed as boxplots or as line charts showing error bars with $\mathrm{SD},{ }^{\star} p<0.05,{ }^{* *} p<0.01$, ${ }^{* *} p<0.001$. All plots $n \geq 3$ independent experiments.

SCD inhibition to a similar degree (Fig. 5B,C). Both neuron and astrocyte populations were also rescued by OLA treatment. Overall, these data show that early ( DIV7) primary cultures are susceptible to SCD inhibition toxicity but that late $(\sim$ DIV18) cultures are resistant.
Having demonstrated that SCD inhibition toxicity was dependent on primary culture age (DIV7 vs DIV18), we then wanted to assess SCD inhibition effects on $\alpha$ Syn-induced cytotoxicity. We treated both early and late cultures with CAY, AAV9- $\alpha$ Syn $3 \mathrm{~K}$ or EV, and OLA for $12 \mathrm{~d}$ 
A
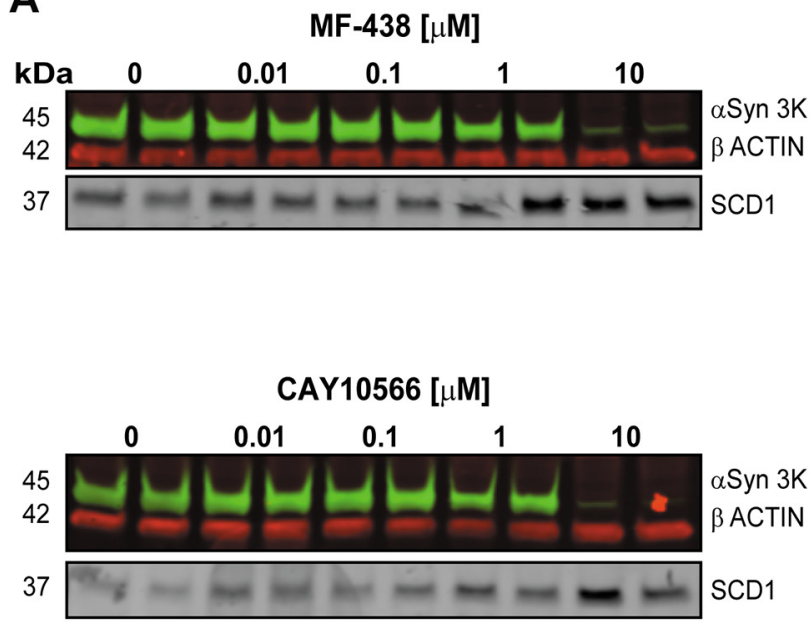

B

$\alpha$ Syn 3K protein

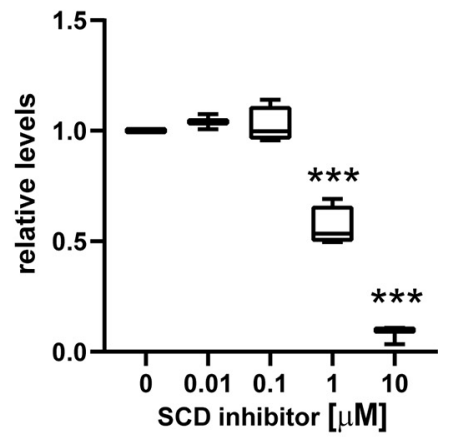

C

aSyn 3K mRNA

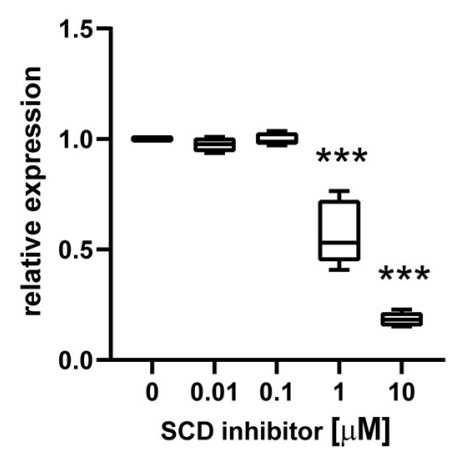

D

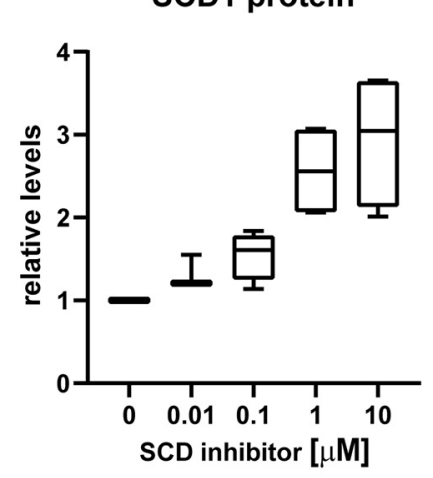

Figure 3. High SCD inhibitor concentrations suppress $\alpha$ Syn 3K-GFP mRNA and protein in neuroblastoma model. $\boldsymbol{A}$, Representative Licor western blot depicting aSyn 3K-GFP, Bactin, and SCD1 protein levels from samples treated with SCD inhibitors (0.01 to 10 $\mu \mathrm{M})$, $\alpha$ Syn 3K-GFP induction and SCD inhibitor treatment was 48 hours before protein isolation. B, Quantification of $\alpha$ Syn $3 K$ protein levels as in $\boldsymbol{A}$, CAY10566 and MF-438 data was equivalent and merged for this plot. $\boldsymbol{C}$, mRNA levels of $\alpha$ Syn 3K-GFP at equivalent time points and treatments as in $\boldsymbol{C}$. Note the drop in $\alpha$ Syn $3 \mathrm{~K}$ protein and mRNA under SCD inhibition $\geq 1 \mu \mathrm{M}$. $\boldsymbol{D}$, Quantification of SCD1 protein levels under increasing concentrations of SCD inhibitor, note the dose dependent increase. Please see Extended Data Figure 3-1 for further SCD mRNA and protein characterization under inhibitor treatment. One-way ANOVA run with Dunnett's multiple test correction, all data displayed as boxplots, ${ }^{* \star *} p<0.001$. All plots $\mathrm{n} \geq 3$ independent experiments.

and then counted neurons and astrocytes (Fig. 5A). Early cultures were more resistant to $\alpha$ Syn $3 \mathrm{~K}$ stress overall ( $\sim 20 \%$ neuron loss vs $\sim 50 \%$ in late neurons) and did not receive any protection from SCD inhibition (as observed before SCD inhibition was toxic; Fig. 5B,C). Although late neuron cultures were more susceptible to $\alpha$ Syn 3K toxicity, they were rescued by SCD inhibition (Fig. 5D,E). Importantly, OLA treatment ablated the SCD inhibitor rescue of $\alpha$ Syn 3K toxicity in late neurons and astrocytes, suggesting the rescue was on-mechanism (Fig. $5 D-F)$. OLA itself exacerbated neuron and astrocyte death under $\alpha$ Syn $3 \mathrm{~K}$ stress in both early and late cultures, supporting its role in promoting $\alpha$ Syn pathology (Fig. 5B-E). Astrocytes showed similar trends to neurons where $\alpha$ Syn $3 \mathrm{~K}$ toxicity was more severe in the late cultures ( $\sim 60 \%$ vs $\sim 90 \%$ astrocyte loss). Viability results with MF was equivalent to CAY (Extended Data Fig. 5-1). Taken together, these data demonstrate that the suppression of MUFAs through SCD inhibition can alleviate $\alpha$ Syn $3 \mathrm{~K}$ stress in late primary neuron cultures but that early cultures receive no protection.

Notably, SCD inhibition by CAY or MF caused an increase in SCD1 mRNA and protein levels in our neuroblastoma model and our primary neuron cultures (Fig. 3; Extended Data Fig. 3-1). The increase in mRNA and protein levels following SCD inhibition was reversed by OLA treatment in both models, suggesting it is a compensatory mechanism. SCD upregulation on its inhibition by small molecules has been observed previously (Vincent et al., 2018). These data further suggest mechanism and robust SCD inhibition by CAY and MF in our in vitro models.

Human iPSC neurons have been shown to tolerate SCD inhibition and even be protected from $\alpha$ Syn toxicity (Vincent et al., 2018; Fanning et al., 2019; Nuber et al., 2021). Based on our differential response to SCD inhibition in our rat primary neuron cultures, we tested early versus late human iPSC neuron cultures under SCD inhibition. Early iPSC neuron cultures started treatment on 

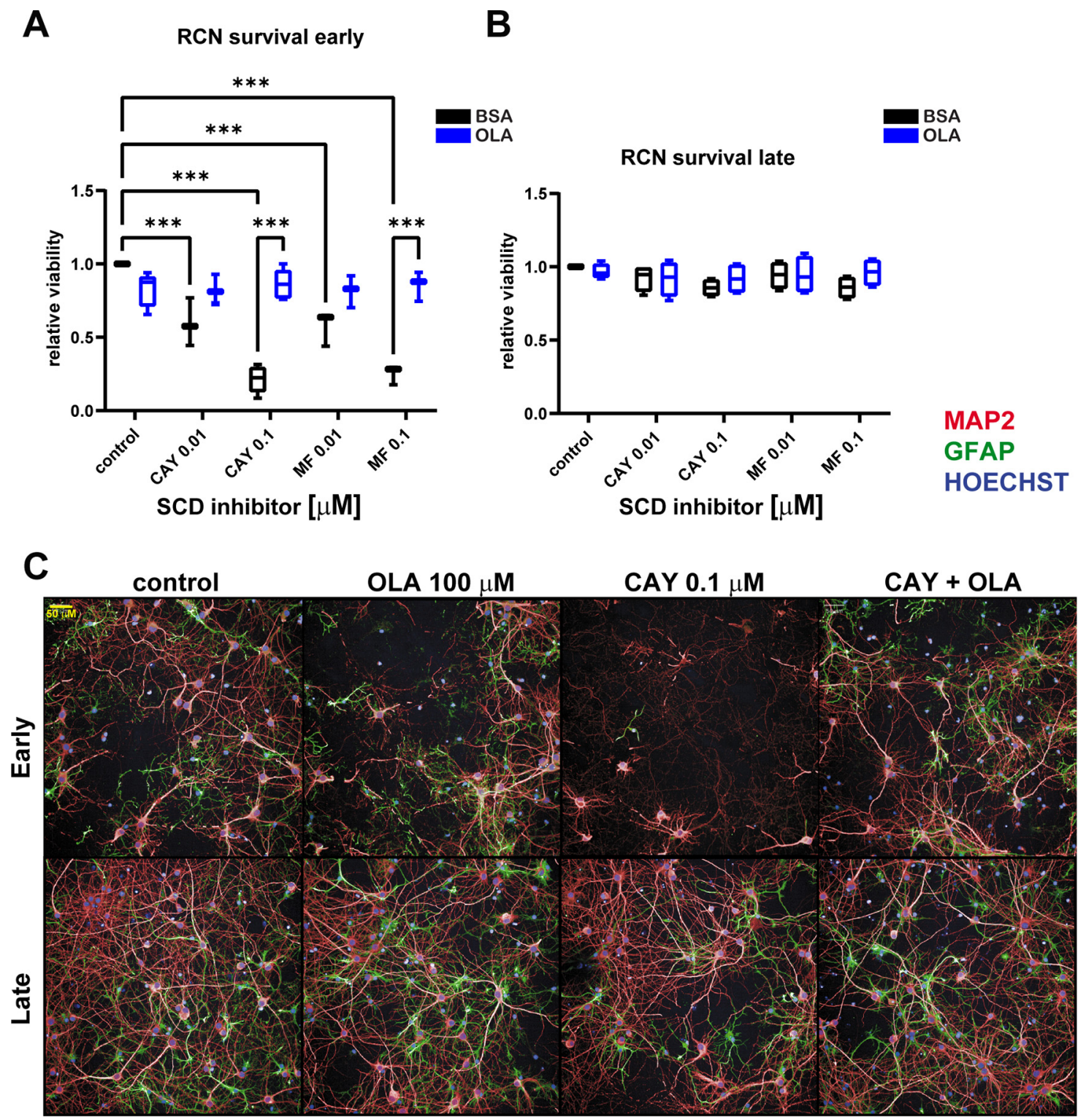

Figure 4. Early primary neuron cultures are sensitive to SCD inhibitor indued toxicity, while late cultures are resistant. $\boldsymbol{A}$, $\boldsymbol{B}$, CellTiter-Glo analysis on primary rat cortical neuron cultures after 12-d treatment \pm SCD inhibitors (0.01 or $0.1 \mu \mathrm{m}) \pm$ OLA (100 $\mu \mathrm{m})$ starting at DIV7 ( $\boldsymbol{A}$, early) or DIV18 (B, late). $\boldsymbol{C}$, Representative high-content images of both early and late cultures as in $\boldsymbol{A}, \boldsymbol{B}$ with OLA, CAY, or both CAY and OLA. Note the SCD inhibitor induced toxicity in early cultures that is rescued by OLA, while there is no toxicity in the late cultures. Two-way ANOVA run with Dunnett's multiple test correction, all data displayed as boxplots, ${ }^{\star \star \star} p<0.001$. All plots $n \geq 3$ independent experiments.

DIV7 and late on DIV21. As before, SCD inhibitors and OLA treatment were done simultaneously, and cultures were assessed for ATP level viability $12 \mathrm{~d}$ later (Fig. 6A). Both early and late iPSC neuron cultures showed dosedependent SCD inhibition-induced cytotoxicity. Similar to our primary neuron cultures, the late iPSC neuron cultures were more resistant to SCD inhibitor-induced toxicity. Late iPSC cultures were resistant up to 0.1 versus $0.01 \mu \mathrm{M}$ for early cultures (Fig. 6B). For example, at $0.1 \mu \mathrm{M}$, early cultures had $\sim 20 \%$ less viability where late cultures had negligible loss on average. OLA rescued SCD inhibition toxicity in the early iPSC neuron cultures but appeared to have minimal effect in the older cultures.
Having established that iPSC human neurons display different sensitivity to SCD inhibition-induced toxicity based on in vitro age, we then compared early and late iPSC neuron cultures under $0.1 \mu \mathrm{m}$ (a concentration that was non-toxic to late but toxic to early) under $\alpha$ Syn $3 \mathrm{~K}$ stress. Even at higher AAV titers than that used in the primary neuron cultures (MOI $250 \mathrm{~K}$ vs $80 \mathrm{~K}$ ), we only observed between $5 \%$ and $20 \%$ ATP loss in the iPSC neurons cultures with late cultures appearing more sensitive to $3 \mathrm{~K}$ stress. Despite the minimal $\alpha$ Syn $3 \mathrm{~K}$-indued neurotoxicity, $0.1 \mu \mathrm{m}$ SCD inhibition could rescue viability in late iPSC neuron cultures (Fig. 6C). OLA treatment reversed this rescue, suggesting on-mechanism protection. 


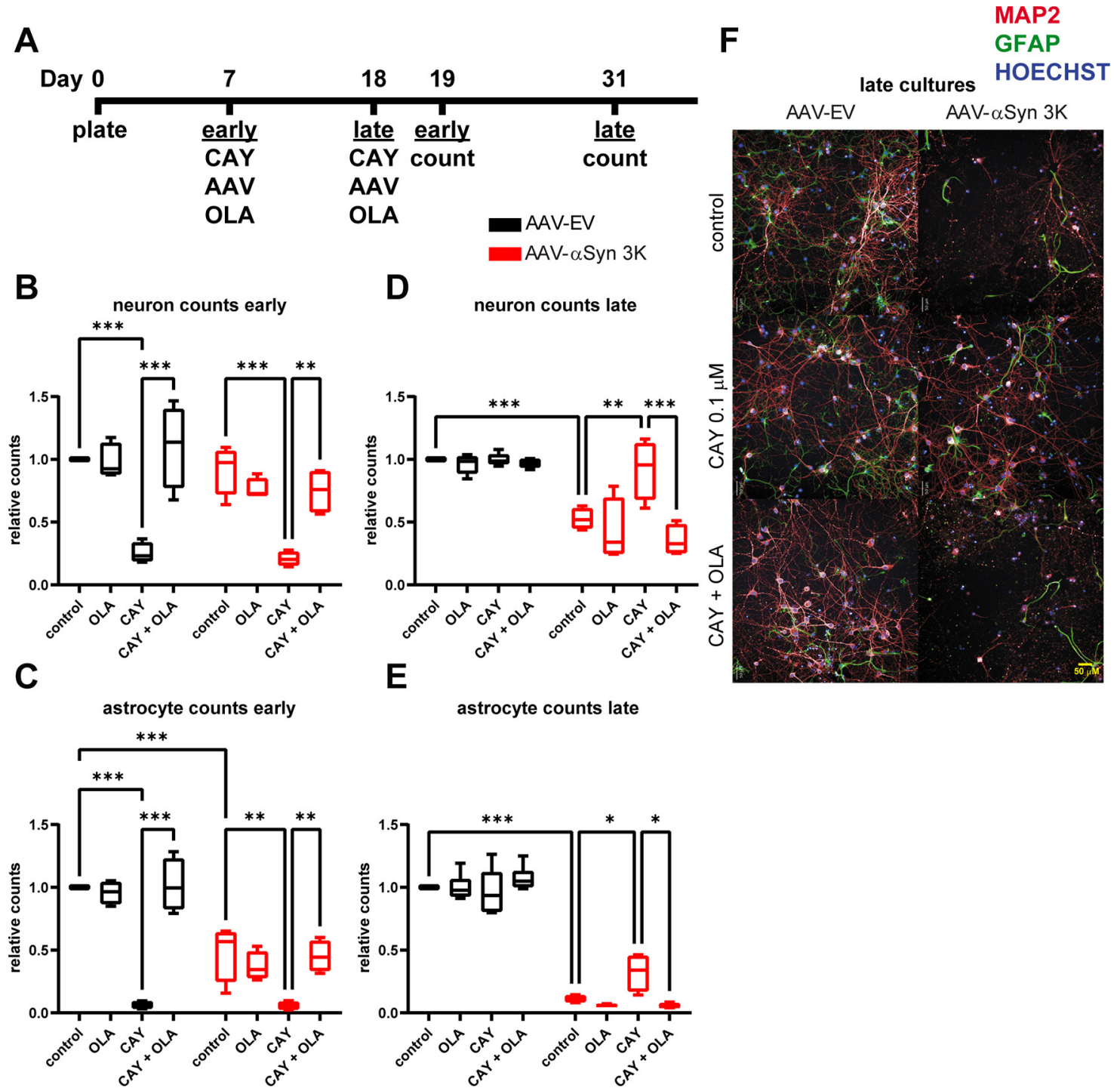

Figure 5. Late primary neuron cultures are protected from $\alpha$ Syn $3 \mathrm{~K}$ toxicity by SCD inhibition (CAY). A, Schematic of treatment schedule in early and late cultures. Primary rat cortical neuron cultures were treated for $12-\mathrm{d} \pm \mathrm{SCD}$ inhibitors $(0.01$ or $0.1 \mu \mathrm{M}) \pm$ OLA $(100 \mu \mathrm{M})+$ AAV9-EV or AAV9- $\alpha$ Syn 3K starting at DIV7 (early) or DIV18 (late). Neurons and astrocytes were counted by staining with MAP2 and GFAP markers, respectively. $\boldsymbol{B}$, Neuron counts from early primary cultures. $\boldsymbol{C}$, Neuron counts from late primary cultures. $\boldsymbol{D}$, Astrocyte counts from early cultures. $\boldsymbol{E}$, Astrocyte counts from late cultures. $\boldsymbol{F}$, Representative high-content images of late cultures as in $\boldsymbol{B}, \boldsymbol{D}$. Note that late cultures are rescued from $\alpha$ Syn 3K toxicity by SCD inhibition, which can be reversed by OLA treatment. Extended Data Figure 5-1 shows results with MF. Two-way ANOVA run with Dunnett's multiple test correction, all data displayed boxplots, ${ }^{*} p<0.05,{ }^{* *} p<0.01,{ }^{* * *} p<0.001$. All plots $n \geq 3$ independent experiments.

As expected, because of their toxicity, SCD inhibitors exacerbated $\alpha$ Syn 3K-induced toxicity in early iPSC neurons (Fig. 6D). Viability results with MF were equivalent to CAY (Extended Data Fig. 6-1). These data demonstrate that the differential response, based on in vitro age, to SCD inhibition-induced toxicity also occurs in human neurons and that non-toxic concentrations can protect neurons from $\alpha$ Syn stress.

\section{Discussion}

We report that a significant factor in primary neuronal susceptibility to toxicity induced by SCD inhibition is their maturity or in vitro age. Our data suggest that there is early dependence on MUFAs and OLA that reduces with culture maturation. This is supported by our finding of early neuron and astrocyte populations being susceptible to SCD inhibition-induced toxicity that can be rescued by OLA treatment (Figs. $4 A, 5 B, C$ ). Late neurons and astrocytes display no significant susceptibility to SCD inhibition toxicity (Figs. $4 B, 5 D, E$ ), presumably because they are less reliant on MUFA synthesis and OLA. Further supporting this differential requirement of MUFAs is that the expression of SCD1 reduces through time in primary neuron cultures, dropping by $\sim 50 \%$ every $6 \mathrm{~d}$ between early and late cultures (Fig. 1D). Furthermore, SCD is a target of 
A

\begin{tabular}{|c|c|c|c|c|}
\hline Day 0 & 7 & 19 & 21 & 33 \\
\hline plate & $\begin{array}{l}\text { early } \\
\text { CAY } \\
\text { AAV } \\
\text { OLA }\end{array}$ & $\frac{\text { early }}{\text { viability }}$ & $\begin{array}{l}\text { late } \\
\text { CAY } \\
\text { AAV } \\
\text { OLA }\end{array}$ & $\frac{\text { late }}{\text { viability }}$ \\
\hline
\end{tabular}

B

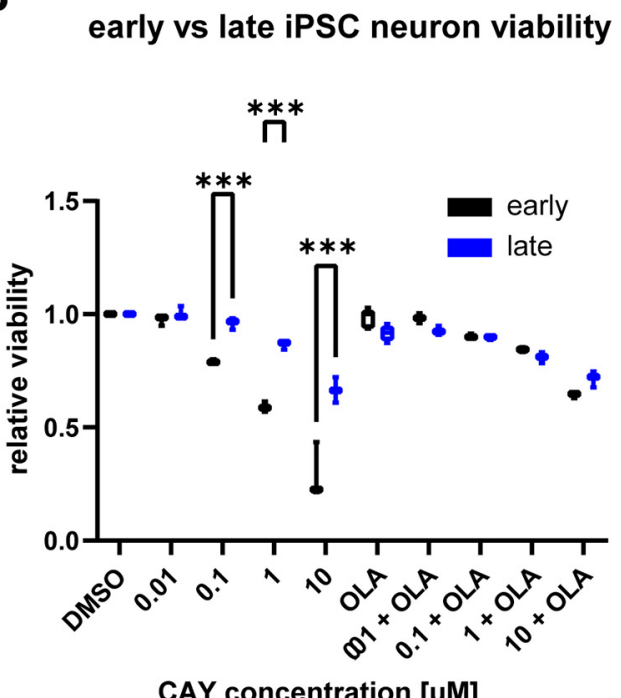

C

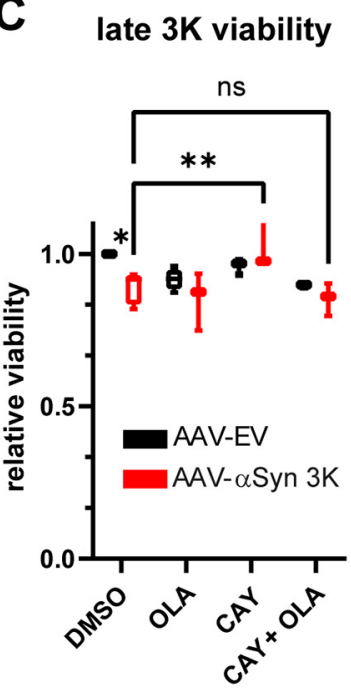

D early $3 K$ viability

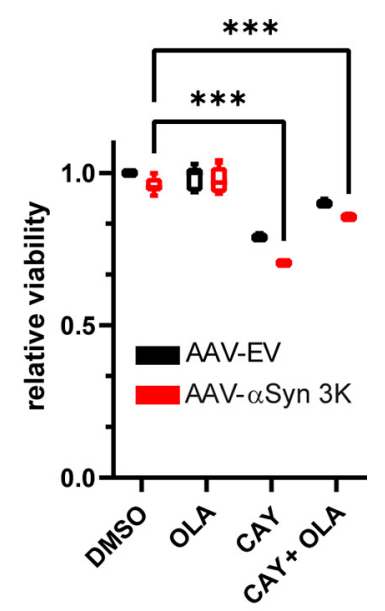

Figure 6. Late human iPSC neurons are protected from $\alpha$ Syn 3K toxicity by SCD inhibition (CAY) and are less sensitive to SCD inhibition toxicity. $\boldsymbol{A}$, Schematic of treatment schedule in early and late cultures. $\boldsymbol{B}$, CellTiter-Glo analysis on human iPSC neuron cultures after $12-d$ treatment \pm CAY $(0.01,0.1,1$, or $10 \mu \mathrm{M}) \pm$ OLA $(10 \mu \mathrm{M})$ starting at DIV7 (early) or DIV21 (late). $\boldsymbol{C}, \boldsymbol{D}$, Same as $\boldsymbol{B}$ but only with $0.1 \mu \mathrm{M}$ CAY and with AAV9-EV or AAV9- $\alpha$ Syn $3 \mathrm{~K}$ for 12-d in late $(\boldsymbol{C})$ or early $(\boldsymbol{D})$ cultures. Note the rescue of viability in late iPSC neuron cultures by $0.1 \mu \mathrm{M}$ CAY (a concentration non-toxic to late neurons but toxic to early neurons). Extended Data Figure 61 shows results with MF, and Extended Data Figure 6-2 shows SCD single-cell expression in human substantia nigra. Two-way ANOVA run with Dunnett's multiple test correction, all data displayed as boxplots, ${ }^{*} p<0.05,{ }^{* *} p<0.01,{ }^{\star \star *} p<0.001$, ns $=$ not significant. All plots $n \geq 3$ independent experiments.

interest in cancer because it is upregulated because of high lipogenic and MUFA demand (from increased metabolic activity and division; Tracz-Gaszewska and Dobrzyn, 2019). A similar mechanism may be at play in early neuron cultures that also have a high metabolic demand and require OLA for synaptic development and neurotrophic support (Medina and Tabernero, 2002; Polo-Hernández et al., 2014) . Any investigator looking to use SCD inhibitors without toxicity side effects in primary neuron models should establish when they no longer induce significant cytotoxicity or when OLA is no longer critical for their survival.

SCD inhibition, when not toxic, rescues $\alpha$ Syn 3K toxicity in primary cultures which can be reversed by OLA treatment (Fig. 5D). OLA treatment itself exacerbated neuron and astrocyte death in our late cultures under $\alpha$ Syn $3 \mathrm{~K}$ stress (Fig. 5D,E). This demonstrates suppression of MUFAs and OLA are key to SCD inhibition-induced protection. OLA levels have specifically been found to be upregulated by excess $\alpha$ Syn and to exacerbate $\alpha$ Syn stress (Fanning et al., 2019). The down regulation of SCD1 and SCD2 (Fig. 1D; Extended Data Fig. 1-1E) under $\alpha$ Syn 3K stress in primary cultures is likely a compensatory process to lower MUFAs and OLA, where significant SCD inhibition accomplishes this and provides protection (as with CAY and MF SCD inhibitors). Conversely, SCD1 was upregulated in our $\alpha$ Syn 3K neuroblastoma model (Fig. 2D). A transformed cell line likely processes $\alpha$ Syn $3 \mathrm{~K}$ stress differently than primary cultures. In either model, the inhibition of SCD and MUFAs is beneficial.

Astrocytes in both the early and late primary cultures were more susceptible to $\alpha$ Syn 3K stress than neurons, this is clear in the early cultures where most death is in the astrocyte population (Fig. 5A,B). OLA synthesized by astrocytes has been shown to be important for neurotrophic support and synaptic development (Medina and Tabernero, 2002; Polo-Hernández et al., 2014). Astrocytes are also reported to have enhanced local translation of SCD and other fatty acid synthesis genes in their peripheral processes (Sakers et al., 2017). It is possible that astrocyte death occurs first from SCD inhibition and promotes neuron death from the loss of OLA and neurotrophic support in early cultures. Mature neuron death from $\alpha$ Syn $3 \mathrm{~K}$ stress may similarly be 
promoted by the dysfunction and death of the astrocyte population. This is not without precedent as, when present, astrocytes can protect dopaminergic neurons from $\alpha$ Syn spreading and aggregation (Tsunemi et al., 2020). Furthermore, it has been shown that excess $\alpha$ Syn increases OLA, triglycerides, and their storage in lipid droplets in neurons (Fanning et al., 2019). Lipid droplets can sequester the build-up of fatty acids, however, neurons generally do not make lipid droplets where astrocytes have an enhanced ability for droplet formation and fatty acid mitigation (Bélanger and Magistretti, 2009). In fact, astrocytes have been shown to protect neurons from toxic fatty acid build-up with detoxification and consumption through B-oxidation after their delivery from neurons (loannou et al., 2019). The interplay of astrocytes and neurons under $\alpha$ Syn 3K stress should be explored further. Additionally, the $\alpha$ Syn 3K model may be useful in the study of synucleinopathies with glial focused pathology such as multiple system atrophy.

Our results with human iPSC neuron cultures were similar to that of our primary rat neuron cultures. Older iPSC neuron cultures were more resistant to SCD inhibition-induced toxicity but did show some sensitivity (Fig. 6B) in contrast to late primary rat neuron cultures which displayed no significant toxicity at the same concentrations. One possible explanation is the that the primary rat neuron cultures are mixed and contain glia, where astrocytes and potentially microglia exert a protective effect. Another being the different SCD isoforms that are present in rodents versus humans, which is discussed below. Additionally, toleration of SCD inhibition has been shown in human iPSC neurons previously but it is unclear at what DIV inhibitors were administered (Vincent et al., 2018; Fanning et al., 2019; Nuber et al., 2021). Presumably in these studies SCD inhibitors were given in primary or iPSC neuron cultures that were matured sufficiently where MUFAs are no longer required for development and survival. To our knowledge this is the first-time showing iPSC neurons are protected specifically from $\alpha$ Syn 3K stress with non-toxic concentrations of SCD inhibitors. Researchers must consider culture age carefully when designing experimental paradigms with SCD inhibitors with in vitro iPSC neuron cultures.

Our independently generated $\alpha$ Syn 3K-GFP accumulation forming cell line confirmed many published results. However, our model showed that high inhibitor concentrations $(\geq 1 \mu \mathrm{M})$ suppressed the expression and protein levels of $\alpha$ Syn 3K-GFP in an off-target manner (Fig. 3). In a similar neuroblastoma model it was found that $\alpha$ Syn $3 \mathrm{~K}$ protein levels were unchanged up to $10 \mu \mathrm{M}$ SCD inhibitor concentrations, although the CAY inhibitor showed a trend of protein lowering (Imberdis et al., 2019). It is important to note that the IC50s of CAY and MF are $\sim 0.005-0.01 \mu \mathrm{m}$ in cell culture (Liu et al., 2007; Uto, 2016) and much higher concentrations are not significantly blocking SCD activity further. Moreover, maximal SCD inhibition at $0.1 \mu \mathrm{m}$ and robust siRNA knock-down of SCD1 both produced $\sim 25 \%$ reduction in accumulations (Fig. 2). Together this suggests that maximal on target suppression of accumulation sy SCD inhibition is $\sim 25 \%$. Interestingly, a $25 \%$ reduction in accumulations fully rescued caspase $3 / 7$ activity, suggesting a threshold for accumulation-induced cytotoxicity. Thus, there may be a smaller true effect of MUFA down regulation (through SCD inhibition) on $\alpha$ Syn $3 \mathrm{~K}$ accumulation formation than previously reported. It is also possible our model's $\alpha$ Syn 3K-GFP expression may be more sensitive to experimental perturbations because of expression construct design or potential parental cell line differences. Despite the issues with high inhibitor concentrations, we found similar results in the suppression of $\alpha$ Syn $3 \mathrm{~K}$ accumulation formation and cytotoxicity with SCD inhibition in our neuroblastoma model.

Rats have two SCD isoforms (SCD1 and SCD2) which share $\sim 87 \%$ sequence homology. In cell culture SCD1 and SCD2 are both inhibited by CAY (Masuda et al., 2012). Taken together, it is likely that both isoforms were blocked by SCD inhibitors in our primary rat neuron culture experiments. Human SCD1 shares $\sim 85 \%$ sequence homology with all rodent SCD isoforms. But the second human SCD isoform, SCD5, is unique to primates, has limited homology to other isoforms, and has high expression in brain tissue (Wang et al., 2005). We examined published single-cell RNA-Seq data from the substantia nigra region in humans (Agarwal et al., 2020) and found SCD5 to be overall higher in human neurons and astrocytes (Extended Data Fig. 6-2), although SCD1 is higher in oligodendrocytes. Yumanity's SCD inhibitor compound YTX-7739, currently in clinical development, targets both SCD1 and SCD5 (Cross, 2018). We believe targeting SCD5 is a good strategy to inhibit SCD activity more effectively in the brain and not elsewhere in the body. This is important because of the connection between SCD and MUFAs to proper immune function and other potential negative side effects of broad SCD inhibition (Meingassner et al., 2013; Zhou et al., 2021).

Out of the top 10 hits in our $\alpha$ Syn 3K toxicity gene expression profiling study, three genes have been previously linked to PD, namely SCD, regulators of G-protein signaling (RGS4) and acyl-CoA synthetase bubblegum family member 1 (ACSBG1). Inhibitors of RGS4 have been shown to reverse D2 antagonist-induced bradykinesia in rats (Blazer et al., 2015), generating interest in their use as novel therapeutics for PD. An RNAi screen discovered that ACSBG1 regulates $\alpha$ Syn levels, which was confirmed in human neurons and mouse brain tissue (Rousseaux et al., 2018). Many of the transcriptional profiling hits were lipid regulatory genes and pathway analysis indeed found several lipid metabolic process enrichments (Extended Data Table 1-2). For instance, sterol regulatory element binding factor 1 (SREBF1) is a transcription factor that regulates SCD and other lipogenic genes. SREBF1 has been identified in a PD genome wide association study and a functional screen for PD (Do et al., 2011; Ivatt et al., 2014). Overall, our transcriptional profiling supports the interconnection between $\alpha$ Syn pathology, MUFAs, and lipids more generally. Investigations of our most significant hit LPCAT1, which is also involved in lipid metabolism, is under way. The fact that many of the 
top gene hits are connected to PD supports the pursuit of investigating LPCAT1 and other novel hits previously unconnected to PD and supports the utility of the $\alpha$ Syn $3 \mathrm{~K}$ model.

Overall, our study supports the use of SCD inhibitors in PD to ameliorate $\alpha$ Syn pathology and neurotoxicity. We demonstrate differential SCD inhibition toxicity based on neuron culture age which must be considered when researching SCD in vitro and has possible clinical implications. It is also important to note that a SCD inhibitor has been assessed in a preclinical mouse model, where it did not induce neurodegeneration and in fact protected from WT and 3K-induced neuron loss (Nuber et al., 2021). Since those who develop PD are overwhelmingly older adults, toxicity to developing or early neurons would not be a major issue; the principal concern is saving established neurons. However, the use of SCD inhibitors in younger people and children with more active neurogenesis should be carefully considered and investigated because of the concerns of neurotoxicity.

\section{References}

Agarwal D, Sandor C, Volpato V, Caffrey TM, Monzón-Sandoval J, Bowden R, Alegre-Abarrategui J, Wade-Martins R, Webber C (2020) A single-cell atlas of the human substantia nigra reveals cell-specific pathways associated with neurological disorders. Nat Commun 11:4183.

Bélanger M, Magistretti PJ (2009) The role of astroglia in neuroprotection. Dialogues Clin Neurosci 11:281-295.

Blazer LL, Storaska AJ, Jutkiewicz EM, Turner EM, Calcagno M, Wade SM, Wang Q, Huang XP, Traynor JR, Husbands SM, Morari M, Neubig RR (2015) Selectivity and anti-Parkinson's potential of thiadiazolidinone RGS4 inhibitors. ACS Chem Neurosci 6:911919.

Courte J, Bousset L, Boxberg YV, Villard C, Melki R, Peyrin JM (2020) The expression level of alpha-synuclein in different neuronal populations is the primary determinant of its prion-like seeding. Sci Rep 10:4895.

Cross R (2018) 2 groups find new Parkinson's target. C\&EN Global Enterprise Vol. 96:4.

Del Carratore F, Jankevics A, Eisinga R, Heskes T, Hong F, Breitling $\mathrm{R}$ (2017) RankProd 2.0: a refactored bioconductor package for detecting differentially expressed features in molecular profiling datasets. Bioinformatics 33:2774-2775.

Dettmer U, Ramalingam N, von Saucken VE, Kim TE, Newman AJ, Terry-Kantor E, Nuber S, Ericsson M, Fanning S, Bartels T, Lindquist S, Levy OA, Selkoe D (2017) Loss of native $\alpha$-synuclein multimerization by strategically mutating its amphipathic helix causes abnormal vesicle interactions in neuronal cells. Hum Mol Genet 26:3466-3481.

Do CB, Tung JY, Dorfman E, Kiefer AK, Drabant EM, Francke U, Mountain JL, Goldman SM, Tanner CM, Langston JW, Wojcicki A, Eriksson N (2011) Web-based genome-wide association study identifies two novel loci and a substantial genetic component for Parkinson's disease. PLoS Genet 7:e1002141.

Dobin A, Davis CA, Schlesinger F, Drenkow J, Zaleski C, Jha S, Batut P, Chaisson M, Gingeras TR (2013) STAR: ultrafast universal RNA-seq aligner. Bioinformatics 29:15-21.

Ericsson M, von Saucken V, Newman AJ, Doehr L, Hoesch C, Kim TE, Dettmer U (2021) Crowded organelles, lipid accumulation, and abnormal membrane tubulation in cellular models of enhanced $\alpha$-synuclein membrane interaction. Brain Res 1758:147349.

Fanning S, Haque A, Imberdis T, Baru V, Barrasa MI, Nuber S, Termine D, Ramalingam N, Ho GPH, Noble T, Sandoe J, Lou Y, Landgraf D, Freyzon Y, Newby G, Soldner F, Terry-Kantor E, Kim
TE, Hofbauer HF, Becuwe M, et al. (2019) Lipidomic analysis of $\alpha$-synuclein neurotoxicity identifies stearoyl CoA desaturase as a target for Parkinson treatment. Mol Cell 73:1001-1014.e8.

Fares MB, Jagannath S, Lashuel HA (2021) Reverse engineering Lewy bodies: how far have we come and how far can we go? Nat Rev Neurosci 22:111-131.

Galvagnion C, Brown JW, Ouberai MM, Flagmeier P, Vendruscolo M, Buell AK, Sparr E, Dobson CM (2016) Chemical properties of lipids strongly affect the kinetics of the membrane-induced aggregation of $\alpha$-synuclein. Proc Natl Acad Sci USA 113:7065-7070.

Imberdis T, Negri J, Ramalingam N, Terry-Kantor E, Ho GPH, Fanning S, Stirtz G, Kim TE, Levy OA, Young-Pearse TL, Selkoe D, Dettmer U (2019) Cell models of lipid-rich $\alpha$-synuclein aggregation validate known modifiers of $\alpha$-synuclein biology and identify stearoyl-CoA desaturase. Proc Natl Acad Sci USA 116:20760-20769.

loannou MS, Jackson J, Sheu SH, Chang CL, Weigel AV, Liu H, Pasolli HA, Xu CS, Pang S, Matthies D, Hess HF, LippincottSchwartz J, Liu Z (2019) Neuron-astrocyte metabolic coupling protects against activity-induced fatty acid toxicity. Cell 177:15221535.e14.

Ivatt RM, Sanchez-Martinez A, Godena VK, Brown S, Ziviani E, Whitworth AJ (2014) Genome-wide RNAi screen identifies the Parkinson disease GWAS risk locus SREBF1 as a regulator of mitophagy. Proc Natl Acad Sci USA 111:8494-8499.

Kaestner KH, Ntambi JM, Kelly TJ Jr, Lane MD (1989) Differentiationinduced gene expression in 3T3-L1 preadipocytes. A second differentially expressed gene encoding stearoyl-CoA desaturase. $J$ Biol Chem 264:14755-14761.

Kubo S, Nemani VM, Chalkley RJ, Anthony MD, Hattori N, Mizuno Y, Edwards RH, Fortin DL (2005) A combinatorial code for the interaction of alpha-synuclein with membranes. J Biol Chem 280:31664-31672.

Li B, Dewey CN (2011) RSEM: accurate transcript quantification from RNA-Seq data with or without a reference genome. BMC Bioinformatics 12:323.

Liu G, Lynch JK, Freeman J, Liu B, Xin Z, Zhao H, Serby MD, Kym PR, Suhar TS, Smith HT, Cao N, Yang R, Janis RS, Krauser JA, Cepa SP, Beno DW, Sham HL, Collins CA, Surowy TK, Camp HS (2007) Discovery of potent, selective, orally bioavailable stearoylCoA desaturase 1 inhibitors. J Med Chem 50:3086-3100.

Love MI, Huber W, Anders S (2014) Moderated estimation of fold change and dispersion for RNA-seq data with DESeq2. Genome Biol 15:550.

Masuda M, Ting TC, Levi M, Saunders SJ, Miyazaki-Anzai S, Miyazaki M (2012) Activating transcription factor 4 regulates stearate-induced vascular calcification. J Lipid Res 53:1543-1552.

Maulik M, Mitra S, Basmayor AM, Lu B, Taylor BE, Bult-Ito A (2019) Genetic silencing of fatty acid desaturases modulates $\alpha$-synuclein toxicity and neuronal loss in Parkinson-like models of $C$. elegans. Front Aging Neurosci 11:207.

Medina JM, Tabernero A (2002) Astrocyte-synthesized oleic acid behaves as a neurotrophic factor for neurons. J Physiol Paris 96:265-271.

Meingassner JG, Aschauer H, Winiski AP, Dales N, Yowe D, Winther MD, Zhang Z, Stütz A, Billich A (2013) Pharmacological inhibition of stearoyl CoA desaturase in the skin induces atrophy of the sebaceous glands. J Invest Dermatol 133:2091-2094.

Nuber S, Rajsombath M, Minakaki G, Winkler J, Müller CP, Ericsson M, Caldarone B, Dettmer U, Selkoe DJ (2018) Abrogating native $\alpha$-synuclein tetramers in mice causes a L-DOPA-responsive motor syndrome closely resembling Parkinson's disease. Neuron 100:75-90.e5.

Nuber S, Nam AY, Rajsombath MM, Cirka H, Hronowski X, Wang J, Hodgetts K, Kalinichenko LS, Müller CP, Lambrecht V, Winkler J, Weihofen A, Imberdis T, Dettmer U, Fanning S, Selkoe DJ (2021) A stearoyl-coenzyme A desaturase inhibitor prevents multiple Parkinson disease phenotypes in $\alpha$-synuclein mice. Ann Neurol 89:74-90.

Polo-Hernández E, Tello V, Arroyo AA, Domínguez-Prieto $M$, de Castro F, Tabernero A, Medina JM (2014) Oleic acid synthesized 
by stearoyl-CoA desaturase (SCD-1) in the lateral periventricular zone of the developing rat brain mediates neuronal growth, migration and the arrangement of prospective synapses. Brain Res 1570:13-25.

Rousseaux MWC, Vázquez-Vélez GE, Al-Ramahi I, Jeong HH, Bajić A, Revelli JP, Ye H, Phan ET, Deger JM, Perez AM, Kim JY, Lavery LA, Xu Q, Li MZ, Kang H, Kim JJ, Shulman JM, Westbrook TF, Elledge SJ, Liu Z, et al. (2018) A druggable genome screen identifies modifiers of $\alpha$-synuclein levels via a tiered cross-species validation approach. J Neurosci 38:9286-9301.

Rovere M, Powers AE, Jiang H, Pitino JC, Fonseca-Ornelas L, Patel DS, Achille A, Langen R, Varkey J, Bartels T (2019) E46K-like $\alpha$-synuclein mutants increase lipid interactions and disrupt membrane selectivity. J Biol Chem 294:9799-9812.

Runwal G, Edwards RH (2021) The membrane interactions of synuclein: physiology and pathology. Annu Rev Pathol 16:465-485.

Sakers K, Lake AM, Khazanchi R, Ouwenga R, Vasek MJ, Dani A, Dougherty JD (2017) Astrocytes locally translate transcripts in their peripheral processes. Proc Natl Acad Sci USA 114:E3830-E3838.

Shahmoradian SH, Lewis AJ, Genoud C, Hench J, Moors TE, Navarro PP, Castaño-Díez D, Schweighauser G, Graff-Meyer A, Goldie KN, Sütterlin R, Huisman E, Ingrassia A, Gier YD, Rozemuller AJM, Wang J, Paepe AD, Erny J, Staempfli A, Hoernschemeyer J, et al. (2019) Lewy pathology in Parkinson's disease consists of crowded organelles and lipid membranes. Nat Neurosci 22:1099-1109.

Sharon R, Goldberg MS, Bar-Josef I, Betensky RA, Shen J, Selkoe DJ (2001) $\alpha$-Synuclein occurs in lipid-rich high molecular weight complexes, binds fatty acids, and shows homology to the fatty acid-binding proteins. Proc Natl Acad Sci USA 98:9110-9115.

Terry-Kantor E, Tripathi A, Imberdis T, LaVoie ZM, Ho GPH, Selkoe D, Fanning S, Ramalingam N, Dettmer U (2020) Rapid alpha-synuclein toxicity in a neural cell model and its rescue by a stearoylCoA desaturase inhibitor. Int J Mol Sci 21:5193.
Yumanity Therapeutics (2021) Yumanity therapeutics provides update on its lead Parkinson's disease clinical program, YTX-7739. Boston: Yumanity Therapeutics Inc.

Tracz-Gaszewska Z, Dobrzyn P (2019) Stearoyl-CoA desaturase 1 as a therapeutic target for the treatment of cancer. Cancers (Basel) 11:948.

Tsunemi T, Ishiguro Y, Yoroisaka A, Valdez C, Miyamoto K, Ishikawa K, Saiki S, Akamatsu W, Hattori N, Krainc D (2020) Astrocytes protect human dopaminergic neurons from $\alpha$-synuclein accumulation and propagation. J Neurosci 40:8618-8628.

Uto $Y$ (2016) Recent progress in the discovery and development of stearoyl CoA desaturase inhibitors. Chem Phys Lipids 197:3-12.

Vincent BM, Tardiff DF, Piotrowski JS, Aron R, Lucas MC, Chung CY, Bacherman H, Chen Y, Pires M, Subramaniam R, Doshi DB, Sadlish H, Raja WK, Solís EJ, Khurana V, Le Bourdonnec B, Scannevin RH, Rhodes KJ (2018) Inhibiting stearoyl-CoA desaturase ameliorates $\alpha$-synuclein cytotoxicity. Cell Rep 25:2742-2754. e31.

Wang J, Yu L, Schmidt RE, Su C, Huang X, Gould K, Cao G (2005) Characterization of HSCD5, a novel human stearoyl-CoA desaturase unique to primates. Biochem Biophys Res Commun 332:735742.

Weihofen A, Liu Y, Arndt JW, Huy C, Quan C, Smith BA, Baeriswyl JL, Cavegn N, Senn L, Su L, Marsh G, Auluck PK, Montrasio F, Nitsch RM, Hirst WD, Cedarbaum JM, Pepinsky RB, Grimm J, Weinreb PH (2019) Development of an aggregate-selective, human-derived $\alpha$-synuclein antibody BIIB054 that ameliorates disease phenotypes in Parkinson's disease models. Neurobiol Dis 124:276-288.

Wingett SW, Andrews S (2018) FastQ Screen: A tool for multi-genome mapping and quality control. F1000Res 7:1338.

Zhou X, Zhu X, Li C, Li Y, Ye Z, Shapiro VS, Copland JA 3rd, Hitosugi T, Bernlohr DA, Sun J, Zeng H (2021) Stearoyl-CoA desaturasemediated monounsaturated fatty acid availability supports humoral immunity. Cell Rep 34:108601. 